\title{
Crystal Plasticity Finite-element Simulation of Ti-6Al-4V Alloy with 3D Polycrystalline Models
}

\author{
Zhi-Hong $\mathrm{WU}^{1, \mathrm{a},{ }^{*}}$, Hong-Chao $\mathrm{KOU}^{1}$, Bin $\mathrm{TANG}^{1}$, \\ Jie $\mathrm{SHAO}^{2}$, Feng-Bo HAN ${ }^{1}$ and Jin-Shan $\mathrm{LI}^{1}$ \\ ${ }^{1}$ State Key Laboratory of Solidification Processing, Northwestern Polytechnical University, Xi'an \\ 710072, China; \\ ${ }^{2}$ AVIC Beijing Aeronautical Manufacturing Technology Research Institute, Beijing 100024, China \\ azhihongwu1992@163.com \\ *Zhi-Hong Wu
}

Keywords: CPFE, Voronoi diagram, Titanium alloy, Stress-strain response

\begin{abstract}
Polycrystalline aggregate models are built based on the theory of Voronoi diagram, then crystal plasticity finite element method (CPFEM) is used to study the relationship of microstructures and stress-strain responses in Ti-6Al-4V alloy during uniaxial tensile tests at room temperature. Stress-strain responses of the Ti-6Al-4V alloy were investigated at different strain rate to study the deformation behavior. Based on the model, the effects of grain size, volume fraction of globular primary $\alpha_{\mathrm{p}}$-phase and strain rate on stress-strain responses are investigated. Results indicate that compared with strain rate and volume fraction of primary $\alpha_{\mathrm{p}}$-phase, grain size show a significant influence on stress-strain responses, the stress has a tendency of increase with the grain refinement. The strain distribution range of aggregates increases with increasing of the strain rate, the stress decreases with increasing of the volume fraction of primary $\alpha_{\mathrm{p}}$-phase.
\end{abstract}

\section{Introduction}

It's well known that titanium and its alloys are widely used as a key structural material in the aviation and space fields due to their superior strength to density ratio, excellent fatigue resistance and corrosion resistance, good biocompatibility and so on. Ti-6Al-4V is a common Ti-Al alloy possess excellent comprehensive performance, and used in a variety of structural engineering applications ranging from medical implants to compressor blades in gas turbine systems[1]. Because of its high strength over density ratio in the low to moderate range of service temperatures, Ti-6Al-4V principally used in the aerospace industry, such as fasteners and engine components[2]. It can be predicted that time dependent material behavior in the range of large strain rate by using material constitutive model. The constitutive law based on crystal plasticity theory can capture the information of both macro and micro deformation behaviors. So finite element simulation technology is a bridge of link the parameters of plastic deformation and the deformation process parameters, reduce the cost and provide production guidance. Taylor and Schmid et al did a lot of meaningful work in the early stages of the crystal plasticity theory development[3]. On the basis of their work, Hill and Rice made the mathematical description focus on Geometry and kinematics of crystal plastic deformation[4,5].

At present, an increasing number of researchers tend to study the deformation process by numerical approaches, such as the crystal plasticity finite element (CPFE) method[6-17]. The CPFE method can be used to predicting the microstructure-property relationships due to its special ability to acquire the details of microstructural features. The geometrical representations of grains is from simple to complex, rough to accurate[18-20]. The incompatibility of plastic deformation in a near $\beta$ titanium alloy was analyzed based on the CPFE method[6], the results show that the plastic deformation in the two phases is incompatible, and the deformation behavior of $\alpha$ phase is significantly affected by the crystal orientation and loading direction. Chung-Hyun Goh et al[7] made a simulation of crystallographic plasticity in fretting of Ti-6Al-4V, results show that the 
crystallographic orientation of the grains plays a significant role in the deformation response. Hamidreza Abdolvand et al[8] used Voronoi tessellation method and crystal plasticity finite element model studied stress heterogeneity within each individual grain of polycrystalline Zircaloy- 2 .

In the present work, a crystal plasticity finite-element model was developed for simulating the uniaxial tensile tests process at room temperature of Ti-6Al-4V alloy with 3D polycrystalline models. The grains was divided by Voronoi polyhedral by regular mesh. The influences of grain size, the volume fraction of primary $\alpha_{\mathrm{p}}$-phase and strain rate on stress-strain responses were investigated in this work.

\section{Model description}

\section{Crystal Plasticity Theory}

Deformation of the crystal can be decomposed into an elastic component and a plastic component. The former include both the elastic distortion and the rigid-body rotation, the latter contain the total deformation caused by crystallographic slip.

$$
\mathbf{F}=\mathbf{F}^{\mathrm{e}} \mathbf{F}^{\mathrm{p}} \text {. }
$$

where $\mathbf{F}^{\mathrm{e}}$ is both the elastic distortion due to lattice distortion and the crystal rigid-body rotation, and $\mathbf{F}^{\mathrm{p}}$ is the plastic deformation due to dislocation slip.

Similarly with multiplicative decomposition of deformation gradient, the velocity gradient is also decomposed a part with elastic distortion and the rigid-body rotation and a part with plastic deformation due to dislocation slip.

$$
\mathbf{L}=\dot{\mathbf{F}} \mathbf{F}^{-1}=\dot{\mathbf{F}}^{\mathrm{e}}\left(\mathbf{F}^{\mathrm{e}}\right)^{-1}+\mathbf{F}^{\mathrm{e}} \dot{\mathbf{F}}^{\mathrm{p}}\left(\mathbf{F}^{\mathrm{p}}\right)^{-1}\left(\mathbf{F}^{\mathrm{e}}\right)^{-1} \text {. }
$$

The plastic velocity gradient $\mathbf{L}^{\mathrm{p}}$ is

$$
\mathbf{L}^{\mathrm{p}}=\dot{\mathbf{F}}^{\mathrm{p}}\left(\mathbf{F}^{\mathbf{p}}\right)^{-1}=\sum_{\boldsymbol{\alpha}} \dot{\gamma}^{\alpha} \mathbf{s}^{\alpha} \otimes \mathbf{m}^{\alpha}
$$

where $\dot{\gamma}^{\alpha}$ is the slipping rate.

The rate-dependent hardening equation is

$$
\dot{\gamma}^{(\alpha)}=\dot{\gamma}_{0}^{(\alpha)}\left[\frac{\tau^{(\alpha)}}{g^{(\alpha)}}\right]\left[\mid\left[\frac{\tau^{(\alpha)}}{g^{(\alpha)}} \mid\right]^{n-1}\right.
$$

where $\dot{\gamma}_{0}^{(\alpha)}$ is the reference shear strain rate, $\mathrm{g}^{(\alpha)}$ is the reference shear stress, and $\mathrm{n}$ the rate sensitivity exponent of slip.

The strain hardening equation is

$$
\dot{g}^{(\alpha)}=\sum_{\beta=1}^{N} h_{\alpha \beta}\left|\dot{\gamma}^{(\beta)}\right|
$$

where $\mathrm{h}_{\alpha \beta}$ is slip hardening moduli.

In 1982, Peirce made the crystal plasticity model numeralization successfully, whereafter Asaro and Needleman come up with the first rate dependent crystal plasticity constitutive relation and the corresponding numerical method. Peirce, Asaro and Needleman[21] proposed a simple form to describe the self-hardening modulus, called PAN model.

$$
h_{\alpha \alpha}=h(\gamma)=h_{0} \operatorname{sech}^{2}\left(\frac{h_{0} \gamma}{\tau_{s}-\tau_{0}}\right) .
$$

where $h_{0}$ represent the initial hardening modulus, $\tau_{0}$ is the initial values of the critical resolved shear stress, $\tau_{\mathrm{s}}$ is saturated flow stress.

\section{Establishment of FE Model}

In recent years, many studies have been focused on simulating polycrystal by using Voronoi diagram. A 3D Voronoi tessellation can be used to establish a model with stochastic grain shape, so more close to actual material structure. Basic conception of Voronoi diagram can be interpreted as a set of randomly distributed core influence zones in the space, and each polyhedron represents a grain. According to the metallographic structure obtained by experimental observation, the grain 
size of Ti-6Al-4V alloy is 15 to $20 \mu \mathrm{m}$. Based on the theory of Voronoi diagram, polycrystalline aggregate models are built with different grain size in this work, grain size are $12 \mu \mathrm{m}, 15 \mu \mathrm{m}$ and 18 $\mu \mathrm{m}$, respectively. A cubic model of scale of $0.07 \mathrm{~mm} * 0.07 \mathrm{~mm} * 0.07 \mathrm{~mm}$ was built, the number of grain are 379, 194 and 112, respectively. Grain morphology is equiaxial, the duplex microstructure containing equiaxed primary $\alpha_{\mathrm{p}}$-phase and lamellar colony structure, the duplex microstructure discrete distribute randomly, the crystallographic orientations with similar probability density distributions are assigned to the finite element mesh. Fig.1 (a) (b) (c) shows the polycrystalline aggregate containing 112,194 and 379 grains.

On the basis of the model built above, Ti-6Al-4V alloy deformation process is simulated with uniaxial tensile tests at different strain rate by using Abaqus finite element simulation software. The boundary conditions of numerical tests are illustrated in Fig.2. Firstly, displacements of three adjacent surfaces of the polycrystalline aggregate are fixed. Then, displacement loading with constant strain rate is applied on the top surface along $\mathrm{Y}$ axis during calculation.
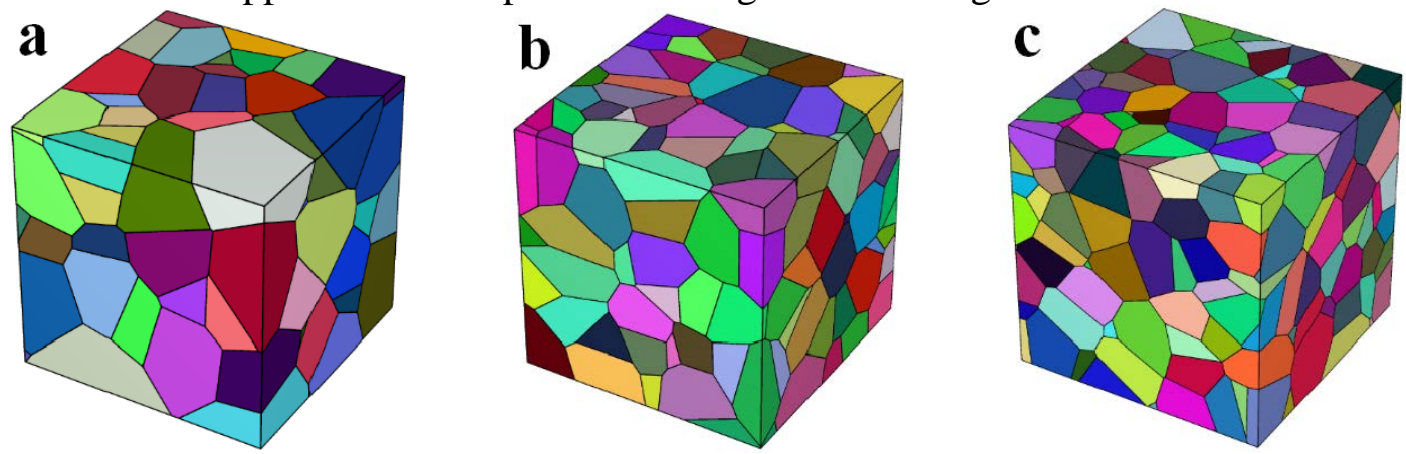

Fig.1 Polycrystalline aggregates with different grain size and boundary conditions of tensile tests.

(a) $18 \mu \mathrm{m}$. (b) $15 \mu \mathrm{m}$. (c) $12 \mu \mathrm{m}$.
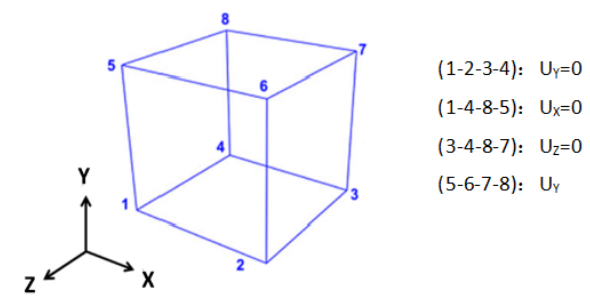

Fig.2 Boundary conditions of tensile tests.

\section{Results and Discussion}

\section{The Material Parameters of the Ti-6Al-4V Alloy}

Ti-6Al-4V alloy is a diphasic titanium alloy, so we need to get the material parameters of alpha and beta phase at room temperature. However, the volume of hexagonal close packed (hcp) $\alpha$-phase is much more than body-centered cubic (bcc) $\beta$-phase, the volume fraction of the $\beta$ phase is rather small. So, The structure of Ti-6Al-4V alloy used in the present model was a duplex microstructure containing globular primary $\alpha_{\mathrm{p}}$-phase nodules and the remaining is a lamellar structure of secondary $\alpha_{\text {s }}$ plates embedded in the $\beta$ matrix[9]. The optical micrograph is shown in Fig.3. The elastic constants of Ti-6Al-4V alloy is $E=110,000 \mathrm{MPa}$, poisson's ratio is $\mu=0.34$. The crystal plasticity parameters of three different slip systems, which are basal, prismatic and pyramidal, of equiaxed primary $\alpha$ grains are listed in Table 1 . The point is that the reference shear rate, inverse strain rate sensitivity exponent and initial values of the yield strength of slip systems are obtained by fitting experimental data use trial-and-error method. The crystal plasticity parameters of lamellar structure in this work is increase in those grains by a factor of 1.25 , e.g $(\alpha+\beta$ colonies $)=1.25$ ( $\alpha_{\mathrm{p}}$ nodulous $)$ [9]. 


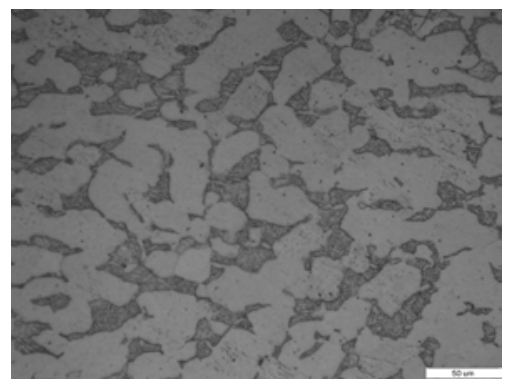

Fig. 3 Microstructure of the Ti-6Al-4V alloy.

Tab. 1 Crystal plasticity constants of Ti-6Al-4V.

\begin{tabular}{cllllll}
\hline Phase and slip system & $\dot{\gamma}_{0}$ & $n$ & $h_{0}(\mathrm{MPa})$ & $\tau_{0}(\mathrm{MPa})$ & $\tau_{s}(\mathrm{MPa})$ \\
\hline \multirow{3}{*}{ lamellar colony } & Basal & 0.001 & 50 & 11853 & 77.5 & 62.5 \\
\cline { 2 - 7 } & Prismatic & 0.001 & 50 & 11853 & 31.3 & 25 \\
\cline { 2 - 7 } & Pyramidal & 0.001 & 50 & 11853 & 65 & 52.5 \\
\hline \multirow{3}{*}{ primary $\alpha_{\mathrm{p}}$-phase } & Basal & 0.001 & 50 & 9482 & 62 & 50 \\
\cline { 2 - 7 } & Prismatic & 0.001 & 50 & 9482 & 25 & 20 \\
\cline { 2 - 7 } & Pyramidal & 0.001 & 50 & 9482 & 52 & 42 \\
\hline
\end{tabular}

\section{Effect of Grain Size on Stress-strain Response.}

Effect of grain size on stress-strain response of aggregates are discussed in this section. The polycrystalline aggregates with grain size of $12 \mu \mathrm{m}, 15 \mu \mathrm{m}$ and $18 \mu \mathrm{m}$ and dispersed with regular mesh contain 3375 elements, and simple uniaxial tensile tests are conducted. If no otherwise specified, 80 per cent of the volume fraction of globular primary $\alpha_{\mathrm{p}}$-phase, regular mesh with 3375 elements and strain rate of $0.001 \mathrm{~s}^{-1}$ are used. Results shown in Fig. 4. It indicates that the percentage of high stress area of the model increase with decreasing of the grain size. We can draw a conclusion that grain size has a significant influence on the overall stress. This is because the number of grain boundary increase with the grain refinement, the proportion of the grain boundary elements increased, stress concentration occurs in the grain boundary elements, which results to higher strength at grain boundary elements. Fig. 5 illustrates the stress-strain curve of aggregates with different grain size during uniaxial tensile tests when the overall strain is 0.1 . It can be seen that the stress has a tendency of increase with the grain refinement. Simulation value is the mean of all integral points of aggregates, hereafter is the same.
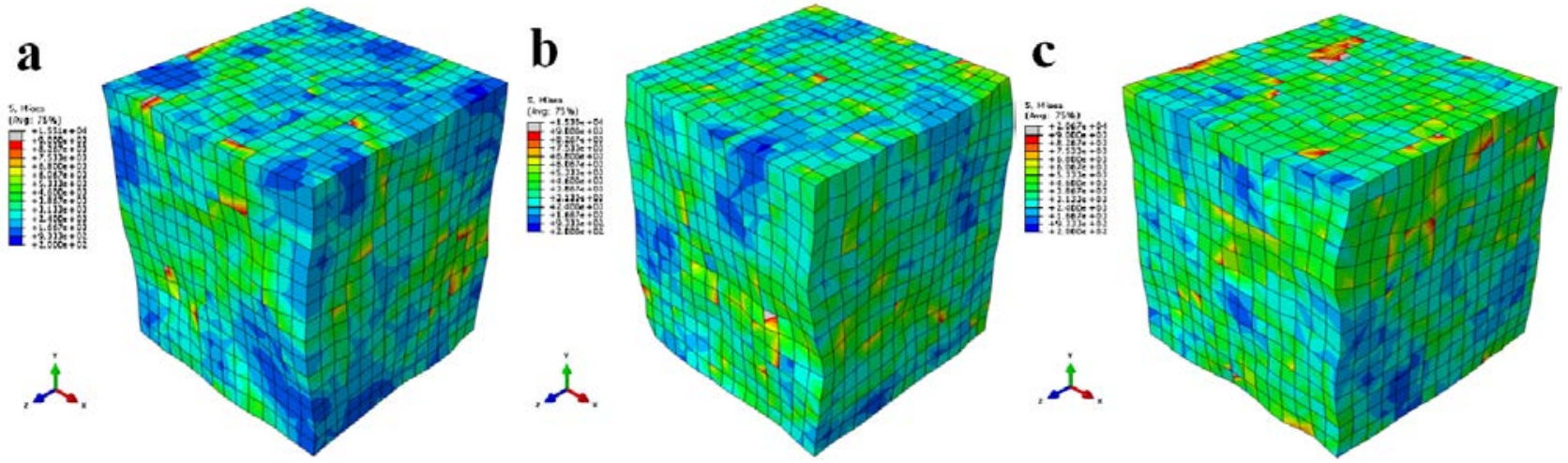

Fig. 4 Distributions of Von Mises equivalent stress of polycrystalline aggregates with different grain size when overall strain is 0.1. (a) $18 \mu \mathrm{m}$. (b) $15 \mu \mathrm{m}$. (c) $12 \mu \mathrm{m}$. 


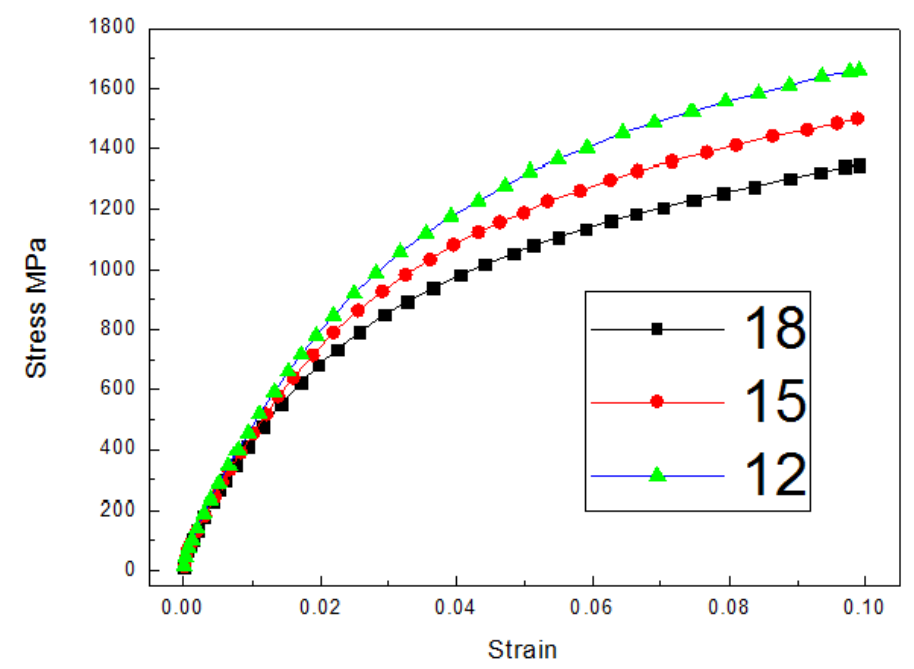

Fig. 5 Stress-strain curves of polycrystalline aggregates with different grain size.

\section{Effect of Strain Rate on Stress-strain Response.}

Fig. 6 illustrates the distributions of strain of aggregates comprised of 112 grains with 3375 hexahedron elements during uniaxial tensile tests when the overall strain is 0.1 and strain rate are $0.001 \mathrm{~s}^{-1}, 0.0002 \mathrm{~s}^{-1}$ and $0.00005 \mathrm{~s}^{-1}$, respectively. From the strain nephogram, the overall strain increases with increasing of the strain rate, this is because force acts on the dislocation instantaneously in higher strain-rate condition, result in dislocation movement speed increase, many of the dislocation source start to move at the same time, besides, twins will appear at high strain rate. For these reasons, metallic materials shows additional hardening with increasing of the strain rate. Fig. 7 shows the stress-strain curve of aggregates calculated by Abaqus using 3375 hexahedron elements with different strain rate when the overall strain is 0.1 . It is noted that strain rate has a slight influence on stress-strain responses.
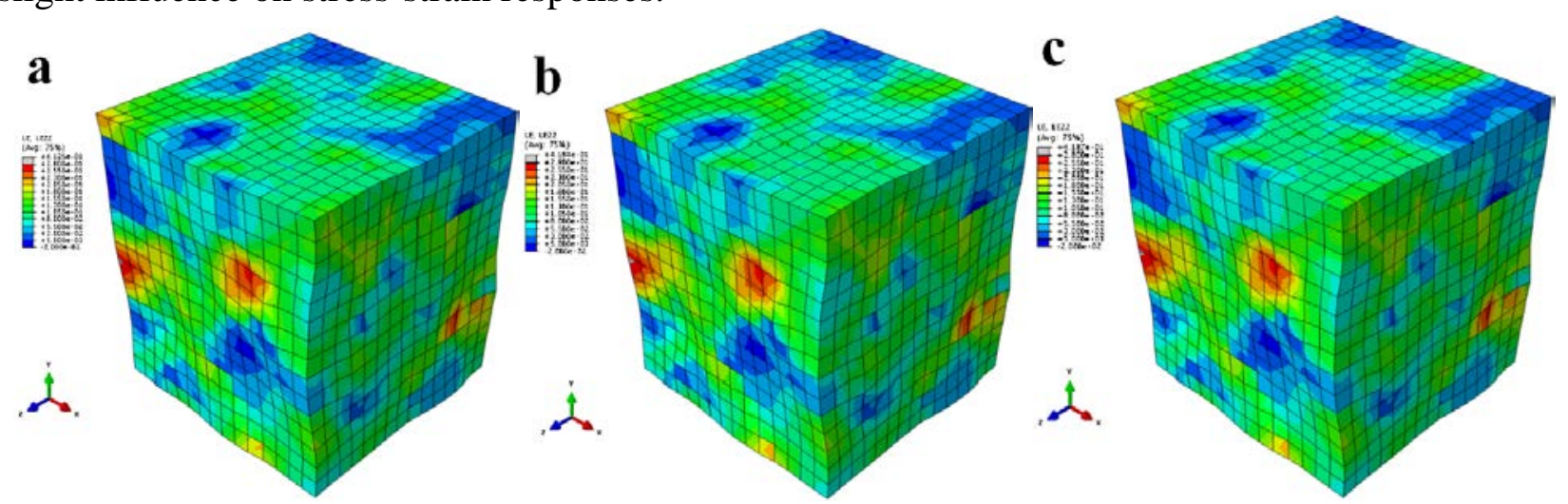

Fig. 6 Distributions of strain of polycrystalline aggregates with different strain rate when overall strain is 0.1 . (a) $0.001 \mathrm{~s}^{-1}$. (b) $0.0002 \mathrm{~s}^{-1}$. (c) $0.00005 \mathrm{~s}^{-1}$. 


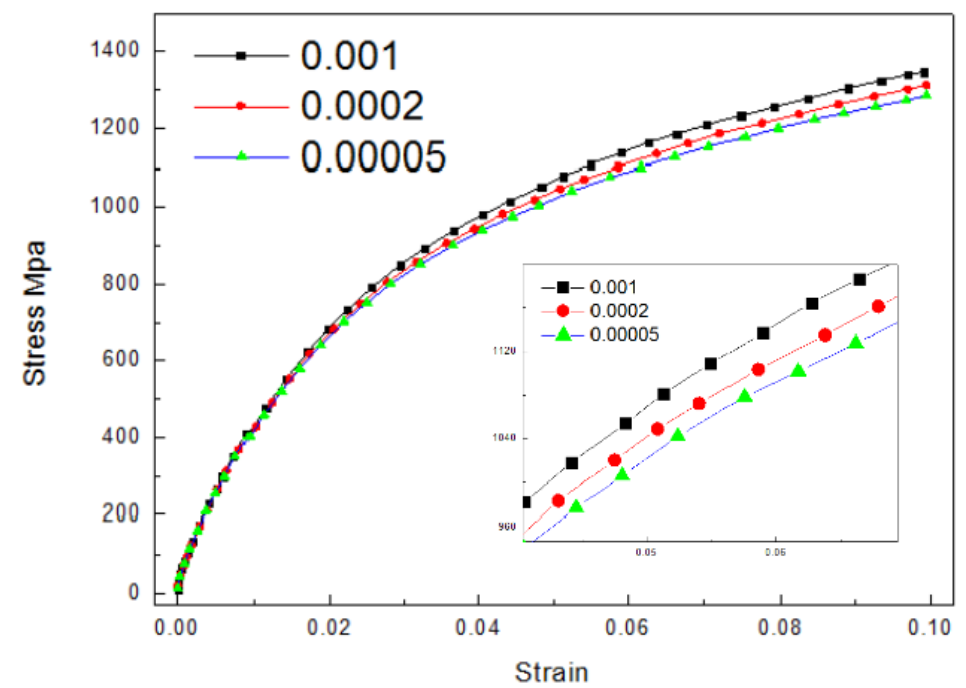

Fig. 7 Stress-strain curves of aggregates with different strain rate.

\section{Effect of the Volume Fraction of Primary $a_{p}$-Phase on Stress-strain Response.}

Fig. 8 presents the Von Mises equivalent stress distribution of aggregates with various volume fraction of primary $\alpha_{\mathrm{p}}$-phase when the overall strain is 0.1 and containing 112 grains with 3375 elements. The volume fraction of primary $\alpha_{\mathrm{p}}$-phase in this section are 20 per cent, 50 per cent and 80 per cent, respectively. From the Fig. 8 , it can be seen that the Von Mises equivalent stress distribution range of aggregates decreases with increasing of the volume fraction of primary $\alpha_{\mathrm{p}}$-phase. The stress distribution of aggregates display primary $\alpha_{\mathrm{p}}$-phase only are shown in Fig. 9. Fig. 10 illustrates the stress-strain curve of aggregates containing 112 grains with different volume fraction of primary $\alpha_{\mathrm{p}}$-phase during uniaxial tensile tests when the overall strain is 0.1 . The conclusion is that the stress decrease slightly with increasing of the primary $\alpha_{\mathrm{p}}$-phase.
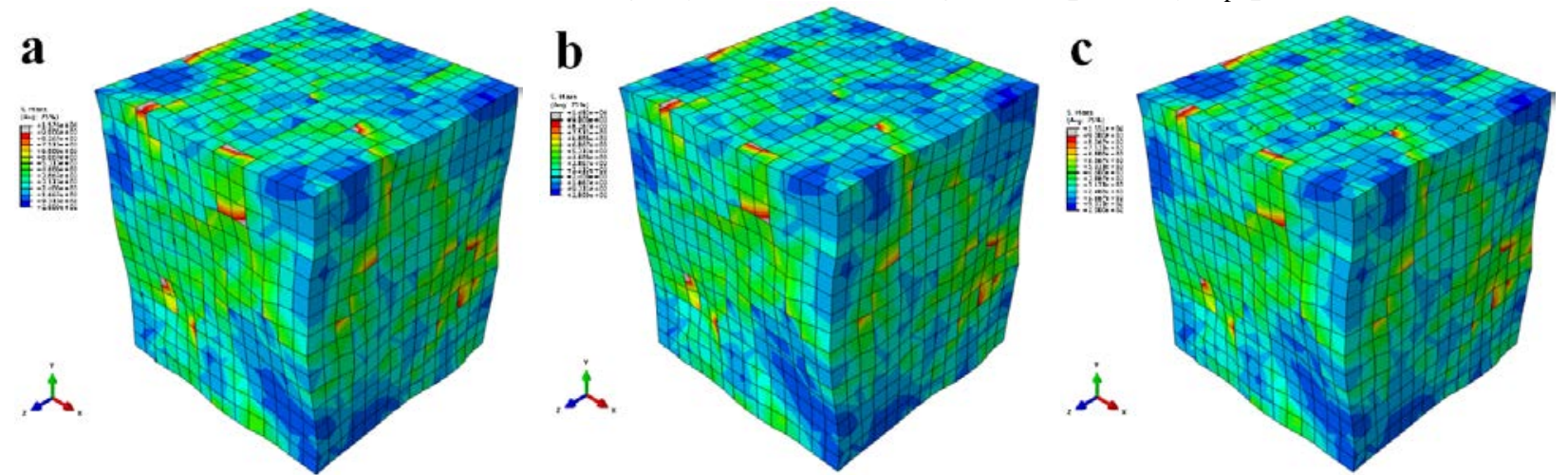

Fig. 8 Distributions of Von Mises equivalent stress of polycrystalline aggregates with different volume fraction of primary $\alpha_{\mathrm{p}}$-phase when overall strain is 0.1 . (a) $20 \%$. (b) $50 \%$. (c) $80 \%$.
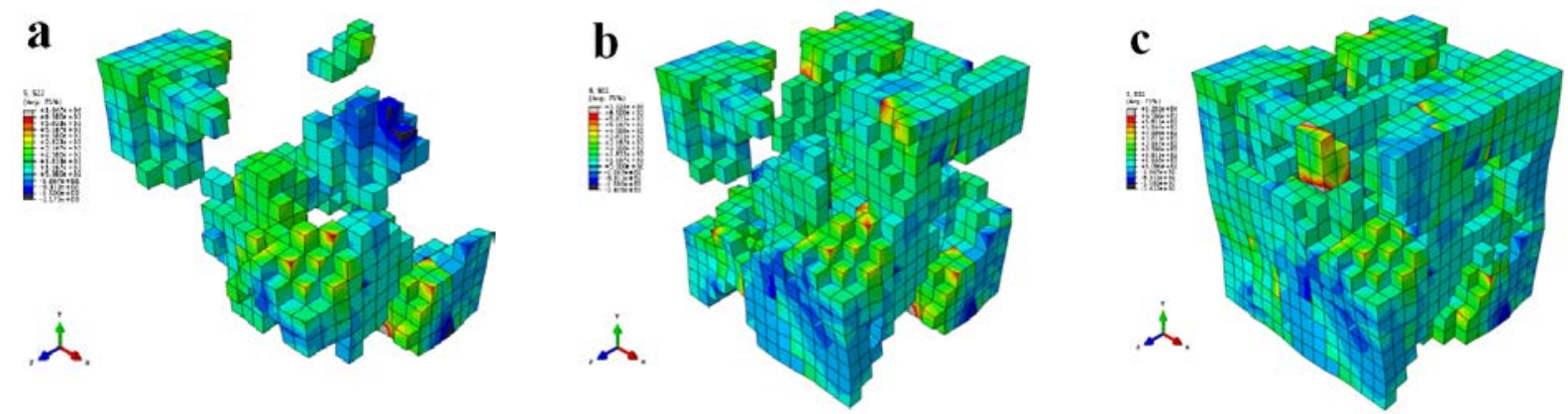

Fig. 9 Distributions of stress of aggregates display primary $\alpha_{\mathrm{p}}$-phase only with different volume fraction of primary $\alpha_{\mathrm{p}}$-phase when overall strain is 0.1 . (a) $20 \%$. (b) $50 \%$. (c) $80 \%$. 


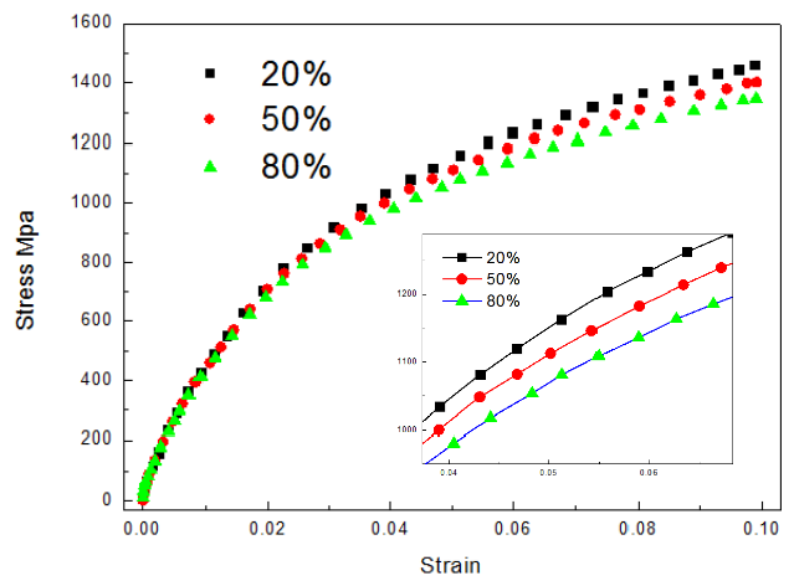

Fig. 10 Stress-strain curves of aggregates with different volume fraction of primary $\alpha_{\mathrm{p}}$-phase.

\section{Conclusions}

1) Based on crystal plasticity finite element simulations, the polycrystalline aggregate models are established by means of Voronoi diagram method in this work. The uniaxial tensile tests process of the Ti-6Al-4V alloy under the condition of constant strain rate was studied. The regular mesh are used to disperse the models. The parameters of the Ti-6Al-4V alloy are got by trial-and-error method.

2) This paper investigated the effects of grain size, the volume fraction of primary $\alpha_{\mathrm{p}}$-phase and strain rate on stress-strain responses.

3) Results show that strain rate has a slight influence on stress-strain responses, whereas the grain size show a significant influence on stress-strain responses. Stress concentration occurs in grain boundaries due to the discontinuity of orientation, the percentage of high stress area increase with grain refinement. The higher strain rate, the higher stress of the polycrystalline aggregate. The stress decreases with increasing of the primary $\alpha_{\mathrm{p}}$-phase.

4) We can see some deficiencies in the model. For example, more perfect grain boundary model still need to build, not considerating complication of the material deformation mechanism, such as twin deformation mechanism. In addition, the superplastic deformation mechanism under high temperature still need to further study. The improved model can provides enhanced understanding of the plastic deformation, especially with regard to the superplastic deformation process.

\section{Acknowledgements}

This work was financially supported by the Natural Science Foundation of Shaanxi Province (2014JQ6216), the Fundamental Research Funds for the Central Universities in China (3102015BJ(

\section{References}

[1] MAYEUR J R, MCDOWELL D L. A three-dimensional crystal plasticity model for duplex Ti-6Al-4V [J]. International Journal of Plasticity, 2007, 23:1457-1485.

[2] V. Tuninetti, G. Gilles, O. Milis, T. Pardoen, A.M. Habraken. Anisotropy and tension-compression asymmetry modeling of the room temperature plastic response of Ti-6Al-4V[J]. International Journal of Plasticity 67 (2015) 53-68.

[3] G. I. Taylor, Plastic strain in metals, J. Inst. Met. 62 (1938) 307-324.

[4] R. Hill, Generalized constitutive relations for incremental deformation of metal crystals by multislip, J. Mech. Phys. Solids. 14 (1966) 95-102. 
[5] J. R. Rice, Inelastic constitutive relations for solids: An internal-variable theory and its application to metal plasticity, J. Mech. Phys. Solids. 19 (1971) 433-455.

[6] Tang Bin, Xie Shao, Liu Yi, Han Fengbo, Kou Hongchao, Li Jinshan. Crystal Plasticity Finite Element Study of Incompatible Deformation Behavior in Two Phase Microstructure in Near $\beta$ Titanium Alloy[J]. Rare Metal Materials and Engineering, 2015, 44(3): 0532-0537.

[7] Chung-Hyun Goh, Richard W.Neu, David L.McDowell. Crystallographic plasticity in fretting of Ti-6Al-4V[J]. International Journal of Plasticity 19 (2003) 1627-1650

[8] Hamidreza Abdolvand,Marta Majkut, Jette Oddershede,Jonathan P. Wright, Mark R. Daymond. Study of 3-D stress development in parent and twin pairs of a hexagonal close-packed polycrystal: Part II-crystal plasticity finite element modeling[J]. Acta Materialia 93 (2015) 235-245

[9] Florent Bridier, David L. McDowell, Patrick Villechaise, José Mendez. Crystal plasticity modeling of slip activity in Ti-6Al-4V under high cycle fatigue loading[J]. International Journal of Plasticity 25 (2009) 1066-1082

[10] Bijish Babu, Lars-Erik Lindgren. Dislocation density based model for plastic deformation and globularization of Ti-6Al-4V[J]. International Journal of Plasticity 50 (2013) 94-108

[11] Masoud Anahid, Mahendra K. Samal, Somnath Ghosh. Dwell fatigue crack nucleation model based on crystal plasticity finite element simulations of polycrystalline titanium alloys[J]. Journal of the Mechanics and Physics of Solids 59 (2011) 2157-2176

[12] M. Zhang, J.Zhang, D.L.McDowell. Microstructure-based crystal plasticity modeling of cyclic deformation of Ti-6Al-4V[J]. International Journal of Plasticity 23 (2007) 1328-1348

[13] J. Lin, Jianbo Yang. GA-based multiple objective optimisation for determining viscoplastic constitutive equations for superplastic alloys[J]. International Journal of Plasticity 15 (1999) 1181-1196

[14] C. Zhang, H. Li, P. Eisenlohr, W. Liu, C.J. Boehlert, M.A. Crimp,T.R. Bieler. Effect of realistic 3D microstructure in crystal plasticity finite element analysis of polycrystalline Ti-5Al-2.5Sn[J]. International Journal of Plasticity 69 (2015) 21-35

[15] Hamidreza Abdolvand, Mark R. Daymond, Charles Mareau. Incorporation of twinning into a crystal plasticity finite element model:Evolution of lattice strains and texture in Zircaloy-2[J]. International Journal of Plasticity 27 (2011) 1721-1738

[16] Z. Zhao, S. Kuchnicki, R. Radovitzky, A. Cuitino. Acta Materialia 55 (2007) 2311-2373.

[17] F. Roters, P. Eisenlohr, L. Hantcherli, D.D. Tjahjanto, T.R. Bieler, D. Raabe. Acta Materialia 58 (2010) 1152 - 1211.

[18] S. Sinha, S. Ghosh, Modeling cyclic ratcheting based fatigue life of HSLA steels using crystal plasticity FEM simulations and experiments, Int. J. Fatigue. 28 (2006) 1690-1704.

[19] L. Delannay, P. J. Jacques, S. R. Kalidindi, Finite element modeling of crystal plasticity with grains shaped as truncated octahedrons, Int. J. Plasticity. 22 (2006) 1879-1898.

[20] O. Diard, S. Leclercq, G. Rousselier, G. Cailletaud, Evaluation of finite element based analysis of 3D multicrystalline aggregates plasticity: Application to crystal plasticity model identification and the study of stress and strain fields near grain boundaries, Int. J. Plasticity. 21 (2005) 691-722.

[21] PEIRCE D, R. ASARO J, NEEDLEMAN A. An analysis of nonuniform and localized deformation in ductile single crystals [J]. Acta Metallurgica, 1982,30:1087-1119. 